\title{
Purity of exponential sums on $\mathbb{A}^{n}$
}

\author{
Antonio Rojas-León
}

\begin{abstract}
We give a purity result for two kinds of exponential sums of the type $\sum_{x \in k^{n}} \psi(f(x))$, where $k$ is a finite field of characteristic $p$ and $\psi: k \rightarrow \mathbb{C}^{\star}$ is a non-trivial additive character. In the first case, $f \in k\left[x_{1}, \ldots, x_{n}\right]$ is a polynomial of degree divisible by $p$ whose highest-degree homogeneous form defines a non-singular projective hypersurface, and in the second case, $f$ is a polynomial of degree prime to $p$ whose highest-degree homogeneous form defines a projective hypersurface with isolated singularities.
\end{abstract}

\section{Introduction}

Let $k$ be a finite field of characteristic $p$ and cardinality $q$, and let $f \in k\left[x_{1}, \ldots, x_{n}\right]$ be a polynomial of degree $d$. Pick a non-trivial additive character $\psi: k \rightarrow \mathbb{C}^{\star}$, and consider the sum $\sum_{x \in k^{n}} \psi(f(x))$. In [Del74], Deligne proved, as a corollary to his proof of the Riemann hypothesis for projective varieties over finite fields, the following estimate.

Theorem 1 [Del74, Théorème 8.4]. Suppose that:

(i) the highest degree homogeneous form $f_{d}$ of $f$ defines a nonsingular hypersurface in $\mathbb{P}_{\bar{k}}^{n-1}$;

(ii) $d$ is prime to $p$.

Then we have the estimate

$$
\left|\sum_{x \in k^{n}} \psi(f(x))\right| \leqslant(d-1)^{n} \cdot q^{n / 2} .
$$

Moreover, he showed that the sum is pure of weight $n$ and rank $(d-1)^{n}$. In particular, there are $(d-1)^{n}$ complex algebraic numbers $\alpha_{1}, \ldots, \alpha_{(d-1)^{n}}$, all pure of weight $n$ (meaning that all their conjugates over $\mathbb{Q}$ have absolute value $q^{n / 2}$ ) such that, for every integer $m \geqslant 1$, if $k_{m}$ denotes the degree $m$ extension of $k$ in a fixed algebraic closure $\bar{k}$, we have

$$
(-1)^{n} \sum_{x \in k_{m}^{n}} \psi\left(\operatorname{Trace}_{k_{m} / k}(f(x))\right)=\sum_{i=1}^{(d-1)^{n}} \alpha_{i}^{m} .
$$

What can we say in the case where $p$ divides $d$ ? By perversity arguments (cf. [KL85, Kat93, Kat05]) we know that the sum is pure for almost all $f \in k\left[x_{1}, \ldots, x_{n}\right]$. More precisely, if we add a sufficiently general linear form to $f$ (one that is contained in a suitable Zariski dense open subset $U$ of the dual affine space $\hat{\mathbb{A}}_{k}^{n}$ depending on $\psi$ and $q$ ), the sum becomes pure of weight $n$. However, these results do not give us any information about the sum associated to a particular $f$. On the other hand, Adolphson and Sperber [AS00b] show, using $p$-adic methods, that if $f$ satisfies certain regularity

Received 2 November 2004, accepted in final form 18 April 2005.

2000 Mathematics Subject Classification 11L03, $11 \mathrm{~L} 07$.

Keywords: exponential sums, $l$-adic cohomology.

Partially supported by MTM2004-07203-C02-01 and FEDER.

This journal is (C) Foundation Compositio Mathematica 2006. 


\section{A. ROJAS-LEÓN}

hypotheses the $L$-function associated to the exponential sum (or its inverse) is a polynomial. In this article we will use these results to give a version of Theorem 1 for the case where $p$ divides $d$.

Fix a prime $\ell \neq p$ and an isomorphism $\iota: \overline{\mathbb{Q}}_{\ell} \rightarrow \mathbb{C}$ so that we can speak about absolute values of elements of $\overline{\mathbb{Q}}_{\ell}$ and weights without ambiguity. From now on we will assume that such an isomorphism has been chosen, without making any further reference to it. Thus, for every $\alpha \in \overline{\mathbb{Q}}_{\ell},|\alpha|$ will always mean $|\iota(\alpha)|$. We will also use this isomorphism to identify the sets of $\mathbb{C}^{\star}$-valued characters

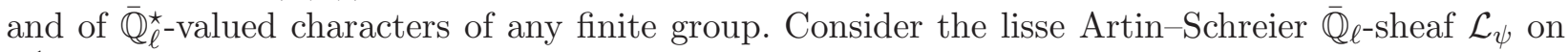
$\mathbb{A}_{k}^{1}$ associated to the non-trivial additive character $\psi: k \rightarrow \mathbb{C}^{\star}$ (cf. [SGA4, Définition 1.7]). For every finite extension $k^{\prime} / k$ and every $t \in \mathbb{A}^{1}\left(k^{\prime}\right)=k^{\prime}$, the trace of the geometric Frobenius element in $\operatorname{Gal}\left(\bar{k} / k^{\prime}\right)$ acting on the stalk of $\mathcal{L}_{\psi}$ at a geometric point $\bar{t}$ over $t$ is $\psi\left(\operatorname{Trace}_{k^{\prime} / k}(t)\right)$. In particular, as $\psi$ takes its values among the roots of unity, $\mathcal{L}_{\psi}$ is pure of weight 0.

Let $\mathcal{L}_{\psi(f)}$ denote the pull-back $f^{\star} \mathcal{L}_{\psi}$ on $\mathbb{A}_{k}^{n}$. The cohomology groups with compact support $\mathrm{H}_{c}^{i}\left(\mathbb{A}_{\bar{k}}^{n}, \mathcal{L}_{\psi(f)}\right)$ are endowed with an action of the absolute Galois group Gal $(\bar{k} / k)$ and, in particular, of the geometric Frobenius element $F \in \operatorname{Gal}(\bar{k} / k)$. By the Grothendieck trace formula we have

$$
\sum_{x \in k^{n}} \psi(f(x))=\sum_{i=0}^{2 n}(-1)^{i} \operatorname{Trace}\left(F \mid \mathrm{H}_{c}^{i}\left(\mathbb{A}_{\bar{k}}^{n}, \mathcal{L}_{\psi(f)}\right)\right) .
$$

Our first result is the following.

Theorem 2. Let $d$ be divisible by $p$. Write $f=f_{d}+f_{d^{\prime}}+f^{\prime}$, where $f_{d}$ is the degree $d$ homogeneous component of $f, d^{\prime}$ is the degree of $f-f_{d}$ and $f_{d^{\prime}}$ is the degree $d^{\prime}$ homogeneous component of $f$. Suppose that:

(a) $d^{\prime} / d>p /\left(p+(p-1)^{2}\right)$ and $d^{\prime}$ is prime to $p$;

(b) the equation $f_{d}=0$ defines a non-singular hypersurface in $\mathbb{P}_{\bar{k}}^{n-1}$;

(c) the hypersurface defined in $\mathbb{P}_{\bar{k}}^{n-1}$ by $f_{d^{\prime}}=0$ does not contain any of the common zeroes of $\partial f_{d} / \partial x_{1}, \ldots, \partial f_{d} / \partial x_{n}$ in $\mathbb{P}_{\bar{k}}^{n-1}$.

Then:

(1) $\mathrm{H}_{c}^{i}\left(\mathbb{A}_{\bar{k}}^{n}, \mathcal{L}_{\psi(f)}\right)=0$ for $i \neq n$;

(2) $\mathrm{H}_{c}^{n}\left(\mathbb{A}_{\bar{k}}^{n}, \mathcal{L}_{\psi(f)}\right)$ has dimension $\left(d^{\prime}(d-1)^{n}+(-1)^{n}\left(d-d^{\prime}\right)\right) / d$ and is pure of weight $n$;

(3) we have the estimate

$$
\left|\sum_{x \in k^{n}} \psi(f(x))\right| \leqslant \frac{d^{\prime}(d-1)^{n}+(-1)^{n}\left(d-d^{\prime}\right)}{d} \cdot q^{n / 2} .
$$

For $d^{\prime}=d-1$ (the generic case) the inequality in part (a) holds as long as $d \geqslant 3$, and we get the following.

Corollary 3. Assume that $d \geqslant 3$ is divisible by p. Let $f=f_{d}+f_{d-1}+f^{\prime}$ be as above. Suppose that:

(a) the equation $f_{d}=0$ defines a non-singular hypersurface in $\mathbb{P}_{\bar{k}}^{n-1}$;

(b) the equation $f_{d-1}=0$ defines a hypersurface in $\mathbb{P}_{\bar{k}}^{n-1}$ that does not contain any of the common zeroes of $\partial f_{d} / \partial x_{1}, \ldots, \partial f_{d} / \partial x_{n}$ in $\mathbb{P}_{\bar{k}}^{n-1}$.

Then:

(1) $\mathrm{H}_{c}^{i}\left(\mathbb{A}_{\bar{k}}^{n}, \mathcal{L}_{\psi(f)}\right)=0$ for $i \neq n$;

(2) $\mathrm{H}_{c}^{n}\left(\mathbb{A}_{\bar{k}}^{n}, \mathcal{L}_{\psi(f)}\right)$ has dimension $\left((d-1)^{n+1}-(-1)^{n+1}\right) / d$ and is pure of weight $n$; 


\section{PURITy OF EXPONENTIAL SUMS ON $\mathbb{A}^{n}$}

(3) we have the estimate

$$
\left|\sum_{x \in k^{n}} \psi(f(x))\right| \leqslant \frac{(d-1)^{n+1}-(-1)^{n+1}}{d} \cdot q^{n / 2} .
$$

As usual, part (3) is a consequence of the vanishing of the cohomology together with Deligne's theorem on weights (cf. [Del80, Corollaire 3.3.4]).

The second result deals with another kind of sum studied by Adolphson and Sperber in [AS00b] and is a generalization of [Gar98, Theorem 0.4]. Let $f \in k\left[x_{1}, \ldots, x_{n}\right]$ be a polynomial of degree $d$, which we will now assume to be prime to $p$. We will show the following.

Theorem 4. Write $f=f_{d}+f_{d^{\prime}}+f^{\prime}$ as in Theorem 2. Suppose that:

(a) $d^{\prime} / d>p /\left(p+(p-1)^{2}\right)$ and $d^{\prime}$ is prime to $p$;

(b) the hypersurface defined by $f_{d}=0$ in $\mathbb{P}_{\bar{k}}^{n-1}$ has at worst weighted homogeneous isolated singularities of total degrees $d_{1}, \ldots, d_{s}$ prime to $p$ (cf. [AS00b, §2], or [Gar98, $\left.\S 0.3\right]$, for the definitions);

(c) the hypersurface defined by $f_{d^{\prime}}=0$ in $\mathbb{P}_{\bar{k}}^{n-1}$ does not contain any of these singularities.

Let $\mu_{1}, \ldots, \mu_{s}$ be the Milnor numbers corresponding to the singularities of $f_{d}=0$. Then:

(1) $\mathrm{H}_{c}^{i}\left(\mathbb{A}_{\bar{k}}^{n}, \mathcal{L}_{\psi(f)}\right)=0$ for $i \neq n$;

(2) $\mathrm{H}_{c}^{n}\left(\mathbb{A}_{\bar{k}}^{n}, \mathcal{L}_{\psi(f)}\right)$ has dimension $(d-1)^{n}-\left(d-d^{\prime}\right) \sum_{i=1}^{s} \mu_{i}$ and is pure of weight $n$;

(3) we have the estimate

$$
\left|\sum_{x \in k^{n}} \psi(f(x))\right| \leqslant\left((d-1)^{n}-\left(d-d^{\prime}\right) \sum_{i=1}^{s} \mu_{i}\right) \cdot q^{n / 2} .
$$

\section{A cohomological vanishing result}

In this section we will begin the proof of Theorem 2. We will first use the method of pencils to show the vanishing of $\mathrm{H}_{c}^{i}\left(\mathbb{A}_{\bar{k}}^{n}, \mathcal{L}_{\psi(f)}\right)$ for $i>n+1$. This requires studying the fibers of the map $f$, so the first thing we need to do is find a suitable compactification of $f$. Unfortunately, the compactification defined in [Kat99] by embedding $\mathbb{A}^{n}$ as a dense open subset of the subscheme of $\mathbb{P}^{n} \times \mathbb{A}^{1}$ given by the vanishing of $F-\lambda X_{0}^{d}$ no longer works in this case. The reason is that we are compactifying a map of degree divisible by $p$, and this may introduce some wild ramification at infinity in the higher direct images of the constant sheaf with respect to the compactified map.

Therefore, instead of directly compactifying $f$, the idea is to first write $f$ as the composition of a closed embedding of $\mathbb{A}^{n}$ in $\mathbb{A}^{n} \times \mathbb{A}^{1}$ (given by the graph of $f$ ) followed by the projection, and then compactify the projection restricted to the image of $\mathbb{A}^{n}$. As we are compactifying a map of degree 1 , we do not run into any problems caused by wild ramification. However, one disadvantage of this compactification is that the fiber at infinity will always have a singular point, so we will only be able to deduce the vanishing of the cohomology groups for $i>n+1$.

Proposition 5. Suppose that the equation $f_{d}=0$ defines a non-singular hypersurface in $\mathbb{P}_{\bar{k}}^{n-1}$. Then $\mathrm{H}_{c}^{i}\left(\mathbb{A}_{\bar{k}}^{n}, \mathcal{L}_{\psi(f)}\right)=0$ for $i>n+1$.

Proof. Define $Z$ to be the hypersurface in $\mathbb{P}_{k}^{n+1}$ (where we take coordinates $X_{0}, \ldots, X_{n}, T$ ) defined by the vanishing of $F-T X_{0}^{d-1}$, where $F$ is the homogenization of $f$ with respect to the variable $X_{0}$ (i.e. $\left.F\left(X_{0}, \ldots, X_{n}\right)=X_{0}^{d} \cdot f\left(X_{1} / X_{0}, \ldots, X_{n} / X_{0}\right)\right)$. The affine space $\mathbb{A}_{k}^{n}$ is naturally an open subscheme of $Z$ (just by embedding it in $\mathbb{A}_{k}^{n+1}$ using the graph of $f$, and then identifying $\mathbb{A}_{k}^{n+1}$ with $\mathbb{P}_{k}^{n+1}$ minus the hyperplane $\left.X_{0}=0\right)$. 


\section{A. ROJAS-LEÓN}

Next, we define the incidence variety $\tilde{Z}$ as a divisor of $Z \times \mathbb{P}_{k}^{1}$, given (with coordinates $X_{0}, \ldots$, $X_{n}, T$ for the first factor and $\lambda_{0}, \lambda_{1}$ for the second factor) by the zero locus of $\lambda_{0} T-\lambda_{1} X_{0}$. Thus,

$$
\tilde{Z}(\bar{k})=\left\{\left(\left(x_{0}, \ldots, x_{n}, t\right),\left(\lambda_{0}, \lambda_{1}\right)\right) \in Z(\bar{k}) \times \mathbb{P}^{1}(\bar{k}): \lambda_{0} t=\lambda_{1} x_{0}\right\} .
$$

Let $\tilde{f}: \tilde{Z} \rightarrow \mathbb{P}_{k}^{1}$ be the restriction to $\tilde{Z}$ of the canonical projection $\pi_{2}: Z \times \mathbb{P}_{k}^{1} \rightarrow \mathbb{P}_{k}^{1}$. It is a proper map, being the composite of a closed immersion and a proper projection (as $Z$ is projective).

The open subset $\mathbb{A}_{k}^{n} \hookrightarrow Z$ can be embedded as an open subscheme of $\tilde{Z}$ in the obvious way. Namely, we identify the point $x \in \mathbb{A}^{n}(\bar{k})$ with $(x, f(x)) \in \tilde{Z}(\bar{k})$. In this way we get a commutative diagram

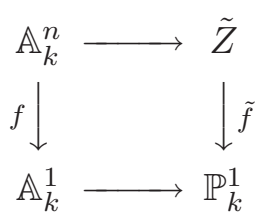

where the horizontal arrows are open embeddings. The image of $\mathbb{A}_{k}^{n}$ in $\tilde{Z}$ can be described as the set of $(x, \lambda) \in \tilde{Z}$ such that $x \notin Z \cap\left\{X_{0}=0\right\}$.

Before going any further we need to show that $\tilde{f}$ is a flat map.

Lemma 6. The map $\tilde{f}: \tilde{Z} \rightarrow \mathbb{P}_{k}^{1}$ is flat.

Proof. By [Har77, Proposition III.9.9] it suffices to show that all geometric fibers of $\tilde{f}$ have the same Hilbert polynomial. The fiber over a finite point $\lambda \in \mathbb{A}^{1}(\bar{k})$ is easily seen to be the complete intersection of the degree $d$ hypersurface $F-\lambda X_{0}^{d}=0$ and the hyperplane $T-\lambda X_{0}=0$. Similarly, the fiber over infinity is the complete intersection of the hypersurface $F=0$ and the hyperplane $X_{0}=0$. As the Hilbert polynomial of a complete intersection only depends on its multidegree, we conclude that it is the same for all geometric fibers of $\tilde{f}$.

We extend by zero the sheaf $\mathcal{L}_{\psi}$ to the whole $\mathbb{P}_{k}^{1}$, and take its pull-back by $\tilde{f}$ to $\tilde{Z}$, which we will also denote by $\mathcal{L}_{\psi(f)}$. This is compatible with the previous notation, as its restriction to $\mathbb{A}_{k}^{n}$ is just the pull-back of $\mathcal{L}_{\psi}$ by $f$.

LEMma 7. There is a quasi-isomorphism

$$
\mathrm{R} \Gamma_{c}\left(\mathbb{A}_{\bar{k}}^{n}, \mathcal{L}_{\psi(f)}\right) \stackrel{\sim}{\rightarrow} \mathrm{R}_{c}\left(\tilde{Z} \otimes \bar{k}, \mathcal{L}_{\psi(f)}\right) .
$$

Proof. To simplify the notation, we will identify each homogeneous form with the projective hypersurface defined by its vanishing. It is clear that $\tilde{Z}_{1}:=\left(Z \cap T \cap X_{0}\right) \times \mathbb{P}_{k}^{1}$ is contained in $\tilde{Z}$ as a closed subscheme. Let $\tilde{Z}_{0}$ be its complement. The restriction of $\tilde{f}$ to $\tilde{Z}_{1}$ is just the second projection. From the decomposition

$$
\tilde{Z}_{0} \stackrel{j}{\hookrightarrow} \tilde{Z} \stackrel{i}{\hookleftarrow} \tilde{Z}_{1}
$$

we get an exact sequence of sheaves

$$
0 \rightarrow j ! j^{\star} \mathcal{L}_{\psi(f)} \rightarrow \mathcal{L}_{\psi(f)} \rightarrow i_{\star} i^{\star} \mathcal{L}_{\psi(f)} \rightarrow 0
$$

from which we get a distinguished triangle in $\mathcal{D}^{b}\left(\overline{\mathbb{Q}}_{\ell}\right.$ - vector spaces $)$

$$
\mathrm{R} \Gamma_{c}\left(\tilde{Z}_{0} \otimes \bar{k}, \mathcal{L}_{\psi(f)}\right) \rightarrow \mathrm{R} \Gamma_{c}\left(\tilde{Z} \otimes \bar{k}, \mathcal{L}_{\psi(f)}\right) \rightarrow \mathrm{R} \Gamma_{c}\left(\tilde{Z}_{1} \otimes \bar{k}, \mathcal{L}_{\psi(f)}\right) \rightarrow .
$$

Now in $\tilde{Z}_{1} \cong\left(Z \cap T \cap X_{0}\right) \times \mathbb{P}_{k}^{1}$ the sheaf $\mathcal{L}_{\psi(f)}$ is just the external tensor product $\overline{\mathbb{Q}}_{\ell} \otimes \mathcal{L}_{\psi}$. Therefore, by the Künneth formula, we have

$$
\mathrm{R} \Gamma_{c}\left(\tilde{Z}_{1} \otimes \bar{k}, \mathcal{L}_{\psi(f)}\right)=\mathrm{R} \Gamma_{c}\left(\left(Z \cap T \cap X_{0}\right) \otimes \bar{k}, \overline{\mathbb{Q}}_{\ell}\right) \otimes \mathrm{R} \Gamma_{c}\left(\mathbb{P}_{\bar{k}}^{1}, \mathcal{L}_{\psi}\right)=0
$$




\section{PURITy OF EXPONENTIAL SUMS ON $\mathbb{A}^{n}$}

as $\mathrm{R} \Gamma_{c}\left(\mathbb{P}_{\bar{k}}^{1}, \mathcal{L}_{\psi}\right)=\mathrm{R} \Gamma_{c}\left(\mathbb{A}_{\bar{k}}, \mathcal{L}_{\psi}\right)=0$ (cf. [SGA4, Théorème $\left.\left.2.7^{*}\right]\right)$. Hence, we get a quasi-isomorphism

$$
\mathrm{R} \Gamma_{c}\left(\tilde{Z}_{0} \otimes \bar{k}, \mathcal{L}_{\psi(f)}\right) \stackrel{\sim}{\rightarrow} \mathrm{R} \Gamma_{c}\left(\tilde{Z} \otimes \bar{k}, \mathcal{L}_{\psi(f)}\right) .
$$

The image of the open immersion $h: \mathbb{A}_{k}^{n} \hookrightarrow \tilde{Z}_{0}$ is the set of $(x, \lambda) \in \tilde{Z}$ such that $x \notin Z \cap X_{0}$. Its complement in $\tilde{Z}_{0}$ is the set of $(x, \lambda) \in \tilde{Z}$ such that $x \in Z \cap X_{0}$ and $x \notin Z \cap T$, so it maps to the point at infinity under $\tilde{f}$. As the stalk of $\mathcal{L}_{\psi}$ at infinity is zero, we have an equality $h_{!} h^{\star} \mathcal{L}_{\psi(f)}=\mathcal{L}_{\psi(f)}$, and therefore a quasi-isomorphism

$$
\mathrm{R} \Gamma_{c}\left(\mathbb{A}_{\bar{k}}^{n}, \mathcal{L}_{\psi(f)}\right) \stackrel{\sim}{\rightarrow} \mathrm{R} \Gamma_{c}\left(\tilde{Z}_{0} \otimes \bar{k}, \mathcal{L}_{\psi(f)}\right) \stackrel{\sim}{\rightarrow} \mathrm{R} \Gamma_{c}\left(\tilde{Z} \otimes \bar{k}, \mathcal{L}_{\psi(f)}\right) .
$$

We will also denote by $\tilde{f}: \tilde{Z} \otimes \bar{k} \rightarrow \mathbb{P}_{\bar{k}}^{1}$ the map deduced from $\tilde{f}: \tilde{Z} \rightarrow \mathbb{P}_{k}^{1}$ by extension of scalars to $\bar{k}$. As $\tilde{f}$ is proper, we have (by composition of derived functors)

$$
\mathrm{R} \Gamma_{c}\left(\tilde{Z} \otimes \bar{k}, \mathcal{L}_{\psi(f)}\right)=\mathrm{R} \Gamma_{c}\left(\mathbb{P}_{\bar{k}}^{1}, \mathrm{R} \tilde{f}_{\star} \mathcal{L}_{\psi(f)}\right) .
$$

On the other hand, by the projection formula we have

$$
\mathrm{R} \tilde{f}_{\star} \mathcal{L}_{\psi(f)}=\mathrm{R} \tilde{f}_{\star}\left(\overline{\mathbb{Q}}_{\ell} \otimes \tilde{f}^{\star} \mathcal{L}_{\psi}\right)=\mathrm{R} \tilde{f}_{\star} \overline{\mathbb{Q}}_{\ell} \otimes \mathcal{L}_{\psi}
$$

so Proposition 5 is equivalent to the following.

Proposition 8. Under the previous hypotheses, the cohomology group $\mathrm{H}_{c}^{i}\left(\mathbb{P}_{\bar{k}}^{1}, \mathrm{R} \tilde{f}_{\star} \overline{\mathbb{Q}}_{\ell} \otimes \mathcal{L}_{\psi}\right)$ vanishes for $i>n+1$.

Therefore, we will prove Proposition 8 instead.

Proposition 9. The sheaves $\mathrm{R}^{i} \tilde{f}_{\star} \overline{\mathbb{Q}}_{\ell}$ on $\mathbb{P}_{\bar{k}}^{1}$ are lisse for $i \geqslant n+1$. For $i=n$ it is the extension of a lisse sheaf by a punctual sheaf.

Proof. The fiber of $\tilde{f}$ at a point $\lambda \in \mathbb{A}^{1}(\bar{k})$ is defined in $\mathbb{P}_{\bar{k}}^{n+1}$ (with the usual coordinates $X_{0}, \ldots$, $\left.X_{n}, T\right)$ by the homogeneous ideal $\left(F-T X_{0}^{d-1}, T-\lambda X_{0}\right)=\left(F-\lambda X_{0}^{d}, T-\lambda X_{0}\right)$. Its intersection with the hyperplane $X_{0}=0$ is then defined by the ideal $\left(F, X_{0}, T\right)$, and is therefore isomorphic to the hypersurface defined in $\mathbb{P}_{\bar{k}}^{n-1}$ by $f_{d}=0$, which is non-singular by hypothesis. Therefore, the fiber itself has at worst isolated singularities. On the other hand, the fiber at $\lambda=\infty$ is defined in $\mathbb{P}_{\bar{k}}^{n+1}$ by the ideal $\left(F, X_{0}\right)$. This is the projective cone over the hypersurface defined in $\mathbb{P}_{\bar{k}}^{n-1}$ by $f_{d}=0$, so it has only one singular point (the vertex).

By [SGA7, Exposé I, Corollaire 4.3] we deduce that for every $\lambda \in \mathbb{P}^{1}(\bar{k})$ the $I_{\lambda}$-invariant specialization map $\left(\mathrm{R}^{i} \tilde{f}_{\star} \overline{\mathbb{Q}}_{\ell}\right)_{\lambda} \rightarrow\left(\mathrm{R}^{i} \tilde{f}_{\star} \overline{\mathbb{Q}}_{\ell}\right)_{\bar{\eta}}$ (where $\bar{\eta}$ is a geometric generic point of $\mathbb{P}_{\bar{k}}^{1}$ and $I_{\lambda}$ the inertia group at $\lambda$ ) is an isomorphism for $i>n$ and surjective for $i=n$. As a consequence, $\mathrm{R}^{i} \tilde{f}_{\star} \overline{\mathbb{Q}}_{\ell}$ is lisse at $\lambda$ for $i>n$. For $i=n$ we have an exact sequence (cf. [Kat99, Theorem 13])

$$
0 \rightarrow(\text { punctual sheaf }) \rightarrow \mathrm{R}^{n} \tilde{f}_{\star} \overline{\mathbb{Q}}_{\ell} \rightarrow j_{\star} j^{\star} \mathrm{R}^{n} \tilde{f}_{\star} \overline{\mathbb{Q}}_{\ell} \rightarrow 0
$$

where $j$ is the inclusion of an open subset of $\mathbb{P}_{\bar{k}}^{1}$ on which $\mathrm{R}^{n} \tilde{f}_{\star} \overline{\mathbb{Q}}_{\ell}$ is lisse. However, as the specialization map $\left(\mathrm{R}^{n} \tilde{f}_{\star} \overline{\mathbb{Q}}_{\ell}\right)_{\lambda} \rightarrow\left(\mathrm{R}^{n} \tilde{f}_{\star} \overline{\mathbb{Q}}_{\ell}\right)_{\bar{\eta}}$ is surjective and $I_{\lambda}$-equivariant, the action of $I_{\lambda}$ on $\left(\mathrm{R}^{n} \tilde{f}_{\star} \overline{\mathbb{Q}}_{\ell}\right)_{\bar{\eta}}$ is trivial. As a consequence, the sheaf $j_{\star} j^{\star} \mathrm{R}^{n} \tilde{f}_{\star} \overline{\mathbb{Q}}_{\ell}$ is lisse at $\lambda$.

Proposition 10. The cohomology group $\mathrm{H}_{c}^{a}\left(\mathbb{P}_{\bar{k}}^{1}, \mathrm{R}^{b} \tilde{f}_{\star} \overline{\mathbb{Q}}_{\ell} \otimes \mathcal{L}_{\psi}\right)$ vanishes for:

(i) $a>2$, all $b$;

(ii) $b>n$, all $a$;

(iii) $b=n, a>0$. 


\section{A. ROJAS-LEÓN}

Proof. Part (i) is clear for cohomological dimension reasons. For $b>n$, the sheaf $\mathrm{R}^{b} \tilde{f}_{\star} \overline{\mathbb{Q}}_{\ell}$ is lisse on $\mathbb{P}_{\bar{k}}^{1}$ by Proposition 9 . As $\mathbb{P}_{\bar{k}}^{1}$ is simply connected, it must be constant. Then, if $\bar{\eta}$ is a geometric generic point of $\mathbb{P}_{\bar{k}}^{1}$, we get

$$
\mathrm{R} \Gamma_{c}\left(\mathbb{P}_{\bar{k}}^{1}, \mathrm{R}^{b} \tilde{f}_{\star} \overline{\mathbb{Q}}_{\ell} \otimes \mathcal{L}_{\psi}\right)=\left(\mathrm{R}^{b} \tilde{f}_{\star} \overline{\mathbb{Q}}_{\ell}\right)_{\bar{\eta}} \otimes \mathrm{R} \Gamma_{c}\left(\mathbb{P}_{\bar{k}}^{1}, \mathcal{L}_{\psi}\right)=0
$$

as $\mathrm{R}_{c}\left(\mathbb{P}_{\bar{k}}^{1}, \mathcal{L}_{\psi}\right)=0$. This proves (ii).

To prove (iii), let $j: V \hookrightarrow \mathbb{P}_{\bar{k}}^{1}$ be as in Proposition 9 , where $V$ is a dense open set on which $\mathrm{R}^{n} \tilde{f}_{\star} \overline{\mathbb{Q}}_{\ell}$ is lisse, and let $\mathcal{H}=j_{\star} j^{\star} \mathrm{R}^{n} \tilde{f}_{\star} \overline{\mathbb{Q}}_{\ell}$. Then $\mathcal{H}$ is lisse on $\mathbb{P}_{\bar{k}}^{1}$ by Proposition 9 , so exactly as above we get $\mathrm{R} \Gamma_{c}\left(\mathbb{P}_{\bar{k}}^{1}, \mathcal{H} \otimes \mathcal{L}_{\psi}\right)=0$. From the exact sequence

$$
0 \rightarrow \mathcal{I}(=\text { punctual sheaf }) \rightarrow \mathrm{R}^{n} \tilde{f}_{\star} \overline{\mathbb{Q}}_{\ell} \rightarrow \mathcal{H} \rightarrow 0
$$

we get, after tensoring with $\mathcal{L}_{\psi}$,

$$
0 \rightarrow \mathcal{I} \otimes \mathcal{L}_{\psi} \rightarrow \mathrm{R}^{n} \tilde{f}_{\star} \overline{\mathbb{Q}}_{\ell} \otimes \mathcal{L}_{\psi} \rightarrow \mathcal{H} \otimes \mathcal{L}_{\psi} \rightarrow 0 .
$$

Now $\mathcal{I} \otimes \mathcal{L}_{\psi}$ is punctual, so $\mathrm{H}_{c}^{i}\left(\mathbb{P}_{\bar{k}}^{1}, \mathcal{I} \otimes \mathcal{L}_{\psi}\right)=0$ for $i>0$. From the long exact sequence of cohomology associated to the exact sequence above we get isomorphisms

$$
\mathrm{H}_{c}^{a}\left(\mathbb{P}_{\bar{k}}^{1}, \mathrm{R}^{n} \tilde{f}_{\star} \overline{\mathbb{Q}}_{\ell} \otimes \mathcal{L}_{\psi}\right) \stackrel{\sim}{\rightarrow} \mathrm{H}_{c}^{a}\left(\mathbb{P}_{\bar{k}}^{1}, \mathcal{H} \otimes \mathcal{L}_{\psi}\right)=0
$$

for $a>0$. This proves (iii).

We can now complete the proof of Proposition 8. We have a spectral sequence

$$
\mathrm{H}_{c}^{a}\left(\mathbb{P}_{\bar{k}}^{1}, \mathrm{R}^{b} \tilde{f}_{\star} \overline{\mathbb{Q}}_{\ell} \otimes \mathcal{L}_{\psi}\right) \Rightarrow \mathrm{H}_{c}^{a+b}\left(\mathbb{P}_{\bar{k}}^{1}, \mathrm{R} \tilde{f}_{\star} \overline{\mathbb{Q}}_{\ell} \otimes \mathcal{L}_{\psi}\right) .
$$

Suppose that $a+b>n+1$. Then either:

- $a>2$, so $\mathrm{H}_{c}^{a}\left(\mathbb{P}_{\bar{k}}^{1}, \mathrm{R}^{b} \tilde{f}_{\star} \overline{\mathbb{Q}}_{\ell} \otimes \mathcal{L}_{\psi}\right)=0$ by part (i) of Proposition 10 ;

- $b>n$, so $\mathrm{H}_{c}^{a}\left(\mathbb{P}_{\bar{k}}^{1}, \mathrm{R}^{b} \tilde{f}_{\star} \overline{\mathbb{Q}}_{\ell} \otimes \mathcal{L}_{\psi}\right)=0$ by part (ii) of Proposition 10 ; or

- $a=2$ and $b=n$, so $\mathrm{H}_{c}^{a}\left(\mathbb{P}_{\bar{k}}^{1}, \mathrm{R}^{b} \tilde{f}_{\star} \overline{\mathbb{Q}}_{\ell} \otimes \mathcal{L}_{\psi}\right)=0$ by part (iii) of Proposition 10.

Therefore, the spectral sequence implies that $\mathrm{H}_{c}^{i}\left(\mathbb{P}_{\bar{k}}^{1}, \mathrm{R} \tilde{f}_{\star} \overline{\mathbb{Q}}_{\ell} \otimes \mathcal{L}_{\psi}\right)$ vanishes for $i>n+1$.

\section{A sum of Milnor numbers computation}

Consider the $L$-function associated to the sheaf $\mathcal{L}_{\psi(f)}$ on $\mathbb{A}_{k}^{n}$ :

$$
L\left(T, \mathcal{L}_{\psi(f)}\right)=\exp \sum_{m=1}^{\infty} \frac{S_{m}}{m} T^{m}
$$

where

$$
S_{m}=\sum_{x \in k_{m}^{n}} \psi\left(\operatorname{Trace}_{k_{m} / k}(f(x))\right)
$$

and $k_{m}$ is the extension of degree $m$ of $k$ in $\bar{k}$. By the Grothendieck trace formula, we have

$$
L\left(T, \mathcal{L}_{\psi(f)}\right)=\prod_{i=0}^{2 n} \operatorname{det}\left(1-T \cdot F \mid \mathrm{H}_{c}^{i}\left(\mathbb{A}_{\bar{k}}^{n}, \mathcal{L}_{\psi(f)}\right)\right)^{(-1)^{i+1}}
$$

where $F \in \operatorname{Gal}(\bar{k} / k)$ is the geometric Frobenius element.

The following result of Adolphson and Sperber [AS00b, Theorem 1.11 and Proposition 6.5] gives an important restriction on the shape of this $L$-function. 


\section{PURITY OF EXPONENTIAL SUMS ON $\mathbb{A}^{n}$}

Theorem 11. Write $f=f_{d}+f_{d^{\prime}}+f^{\prime}$, where $f_{d}$ is the degree $d$ homogeneous component of $f$, $d^{\prime}$ is the degree of $f-f_{d}$ and $f_{d^{\prime}}$ is the degree $d^{\prime}$ homogeneous component of $f$. Suppose that $d^{\prime} / d>p /\left(p+(p-1)^{2}\right)$ and $d^{\prime}$ is prime to $p$. Suppose also that $\partial f_{d} / \partial x_{1}, \ldots, \partial f_{d} / \partial x_{n}$ have a finite number of common zeroes in $\mathbb{P}_{\bar{k}}^{n-1}$ (which is automatic if the hypersurface $f_{d}=0$ in $\mathbb{P}_{\bar{k}}^{n-1}$ is nonsingular) and the hypersurface defined in $\mathbb{P}_{\bar{k}}^{n-1}$ by $f_{d^{\prime}}=0$ does not contain any of them. Then $L\left(T, \mathcal{L}_{\psi(f)}\right)^{(-1)^{n+1}}$ is a polynomial of degree $(d-1)^{n}-\left(d-d^{\prime}\right) \sum_{i=1}^{s} \mu_{i}$, where the sum is taken over the set $\left\{P_{1}, \ldots, P_{s}\right\}$ of common zeroes of $\partial f_{d} / \partial x_{1}, \ldots, \partial f_{d} / \partial x_{n}$ in $\mathbb{P}_{\bar{k}}^{n-1}$ and $\mu_{i}$ denotes the corresponding Milnor number

$$
\mu_{i}=\operatorname{dim}_{\bar{k}} \mathcal{O}_{S, P_{i}} .
$$

Here $S$ is the zero-dimensional subscheme of $\mathbb{P}_{\bar{k}}^{n-1}$ defined by the ideal $\left(\partial f_{d} / \partial x_{1}, \ldots, \partial f_{d} / \partial x_{n}\right)$, and $\mathcal{O}_{S, P_{i}}$ its local ring at $P_{i}$, which is a finite $\bar{k}$-algebra.

We will now compute this sum of Milnor numbers explicitly in the following more general setting. Lemma 12. Let $F_{1}, \ldots, F_{n} \in \bar{k}\left[x_{1}, \ldots, x_{n}\right]$ be (possibly zero) homogeneous polynomials of degree d-1. Suppose that:

(i) $F_{1}, \ldots, F_{n}$ have a finite number of common zeroes in $\mathbb{P}_{\bar{k}}^{n-1}$;

(ii) we have the relation

$$
\sum_{i=1}^{n} x_{i} \cdot F_{i}=0 .
$$

Let $\left\{P_{1}, \ldots, P_{s}\right\}$ be the set of common zeroes of $F_{1}, \ldots, F_{n}$ in $\mathbb{P}_{\bar{k}}^{n-1}$, and for every $i=1, \ldots, s$ let $\mu_{i}$ be the corresponding Milnor number. Then we have

$$
\sum_{i=1}^{s} \mu_{i}=\frac{(d-1)^{n}-(-1)^{n}}{d}
$$

Proof. By induction on $n$, we first prove it for $n=2$. In this case, both $F_{1}$ and $F_{2}$ must be non-zero (otherwise, by (ii) they would both be zero, and (i) would not hold). The relation $x_{1} F_{1}+x_{2} F_{2}=0$ implies that $x_{1}$ divides $F_{2}$ and $x_{2}$ divides $F_{1}$. Let $F_{1}=x_{2} G_{1}$ and $F_{2}=x_{1} G_{2}$. Then $x_{1} x_{2}\left(G_{1}+G_{2}\right)=$ 0 , so $G_{2}=-G_{1}$. Therefore, the subscheme defined by $F_{1}$ and $F_{2}$ is that defined by $G_{1}$, which is a polynomial of degree $d-2$. The common zeroes of $F_{1}$ and $F_{2}$ are then in one-to-one correspondence with the distinct linear factors of $G_{1}$, and the Milnor numbers are the corresponding multiplicities. Thus, in this case we get $\sum_{i=1}^{s} \mu_{i}=d-2=\left((d-1)^{2}-1\right) / d$.

We assume now that the lemma is true for $n-1 \geqslant 2$, and prove it for $n$. Choose $\left(\alpha_{1}, \ldots, \alpha_{n-1}\right) \in$ $\bar{k}^{n-1}$ such that none of the points $P_{1}, \ldots, P_{s}$ is contained in the hyperplane $x_{n}-\sum_{j=1}^{n-1} \alpha_{j} x_{j}=0$. We construct the polynomials $F_{1}^{\prime}, \ldots, F_{n}^{\prime}$ given by

$$
\begin{aligned}
F_{i}^{\prime}\left(x_{1}, \ldots, x_{n-1}, x_{n}\right)= & F_{i}\left(x_{1}, \ldots, x_{n-1}, x_{n}+\sum_{j=1}^{n-1} \alpha_{j} x_{j}\right) \\
& +\alpha_{i} F_{n}\left(x_{1}, \ldots, x_{n-1}, x_{n}+\sum_{j=1}^{n-1} \alpha_{j} x_{j}\right) \quad \text { for } i=1, \ldots, n-1 \\
F_{n}^{\prime}\left(x_{1}, \ldots, x_{n-1}, x_{n}\right)= & F_{n}\left(x_{1}, \ldots, x_{n-1}, x_{n}+\sum_{j=1}^{n-1} \alpha_{j} x_{j}\right) .
\end{aligned}
$$




\section{A. ROJAS-LEÓN}

Then the schemes $S$ defined by the ideal $\left(F_{1}, \ldots, F_{n}\right)$ and $S_{1}$ defined by $\left(F_{1}^{\prime}, \ldots, F_{n}^{\prime}\right)$ correspond to each other via the automorphism $\varphi$ of $\mathbb{P}_{\bar{k}}^{n-1}$ given by $\varphi\left(x_{1}, \ldots, x_{n-1}, x_{n}\right)=\left(x_{1}, \ldots, x_{n-1}, x_{n}+\right.$ $\left.\sum_{j=1}^{n-1} \alpha_{j} x_{j}\right)$. In particular, the sums of the Milnor numbers at the points of $S$ and $S_{1}$ are the same. Moreover, we have

$$
\begin{aligned}
\sum_{i=1}^{n} x_{i} \cdot F_{i}^{\prime}\left(x_{1}, \ldots, x_{n-1}, x_{n}\right)= & \sum_{i=1}^{n-1} x_{i} \cdot\left(F_{i}\left(x_{1}, \ldots, x_{n-1}, x_{n}+\sum_{j=1}^{n-1} \alpha_{j} x_{j}\right)\right. \\
& \left.+\alpha_{i} F_{n}\left(x_{1}, \ldots, x_{n-1}, x_{n}+\sum_{j=1}^{n-1} \alpha_{j} x_{j}\right)\right) \\
& +x_{n} \cdot F_{n}\left(x_{1}, \ldots, x_{n-1}, x_{n}+\sum_{j=1}^{n-1} \alpha_{j} x_{j}\right) \\
= & \sum_{i=1}^{n-1} x_{i} \cdot F_{i}\left(x_{1}, \ldots, x_{n-1}, x_{n}+\sum_{j=1}^{n-1} \alpha_{j} x_{j}\right) \\
& +\left(x_{n}+\sum_{i=1}^{n-1} \alpha_{i} x_{i}\right) \cdot F_{n}\left(x_{1}, \ldots, x_{n-1}, x_{n}+\sum_{j=1}^{n-1} \alpha_{j} x_{j}\right)=0 .
\end{aligned}
$$

If $P=\left(x_{1}, \ldots, x_{n}\right)$ is a common zero of $F_{1}^{\prime}, \ldots, F_{n}^{\prime}$, then $\varphi(P)=\left(x_{1}, \ldots, x_{n-1}, x_{n}+\sum_{j=1}^{n-1} \alpha_{j} x_{j}\right)$ is a common zero of $F_{1}, \ldots, F_{n}$ so, by the choice of the $\alpha_{i}, \varphi(P)$ is not contained in the hyperplane $x_{n}-\sum_{j=1}^{n-1} \alpha_{j} x_{j}=0$. Hence, $P$ is not contained in the hyperplane $x_{n}=0$. Therefore, we can, and do, assume that none of the common zeroes of $F_{1}, \ldots, F_{n}$ is contained in the hyperplane $x_{n}=0$.

Under this assumption, we claim that $F_{1}, \ldots, F_{n-1}$ form a regular sequence in $\bar{k}\left[x_{1}, \ldots, x_{n}\right]$ (compare [AS00b, Lemma 5.1]). Otherwise, the subscheme defined by them in $\mathbb{P}_{\bar{k}}^{n-1}$ would have an irreducible component $Y$ of dimension at least 1. From (ii) we deduce that $Y$ is contained in the hypersurface $x_{n} F_{n}=0$. Being irreducible, it must be contained either in $x_{n}=0$ or in $F_{n}=0$. Furthermore, as it has dimension $\geqslant 1$, its intersections with both $x_{n}=0$ and $F_{n}=0$ are nonempty. So in either case, the intersection of $F_{1}, \ldots, F_{n-1}, F_{n}$ and $x_{n}=0$ would be non-empty, in contradiction with the assumption made above.

Denote by $S_{1}$ the subscheme of $\mathbb{P}_{\bar{k}}^{n-1}$ defined by $\left(F_{1}, \ldots, F_{n-1}\right)$. The support of $S_{1}$ is the disjoint union of the points $P_{1}, \ldots, P_{s}$, which are contained in $F_{n}=0$, and the points $P_{s+1}, \ldots, P_{s+r}$, which are contained in $x_{n}=0$. Let $\nu_{1}, \ldots, \nu_{s+r}$ be the corresponding Milnor numbers (i.e. $\nu_{i}=$ $\left.\operatorname{dim}_{\bar{k}} \mathcal{O}_{S_{1}, P_{i}}\right)$. As $F_{1}, \ldots, F_{n-1}$ form a regular sequence of polynomials of degree $d-1, S_{1}$ is a zerodimensional complete intersection of degree $(d-1)^{n-1}$, therefore

$$
\sum_{i=1}^{s+r} \nu_{i}=\operatorname{dim}_{\bar{k}} \Gamma\left(S_{1}, \mathcal{O}_{S_{1}}\right)=(d-1)^{n-1} .
$$

For every $i=1, \ldots, s, x_{n}$ is invertible in the local $\operatorname{ring} \mathcal{O}_{\mathbb{P}^{n-1}, P_{i}}$. So from (ii) we deduce that $F_{n}$ is contained in the ideal generated by $F_{1}, \ldots, F_{n-1}$ in this local ring. Therefore

$$
\mathcal{O}_{S, P_{i}}=\mathcal{O}_{\mathbb{P}^{n-1}, P_{i}} /\left(F_{1}, \ldots, F_{n-1}, F_{n}\right)=\mathcal{O}_{\mathbb{P}^{n-1}, P_{i}} /\left(F_{1}, \ldots, F_{n-1}\right)=\mathcal{O}_{S_{1}, P_{i}}
$$

and, in particular, $\nu_{i}=\mu_{i}$.

On the other hand, for $i=1, \ldots, r, F_{n}$ is invertible in the local ring $\mathcal{O}_{\mathbb{P} n-1}, P_{s+i}$, so $x_{n}$ is contained in the ideal generated by $F_{1}, \ldots, F_{n-1}$ in this local ring. Let $G_{j}=F_{j}\left(x_{1}, \ldots, x_{n-1}, 0\right)$, $S_{2}$ the subscheme of $\mathbb{P}_{\bar{k}}^{n-2}$ (which we identify with the hyperplane $x_{n}=0$ in $\mathbb{P}_{\bar{k}}^{n-1}$ ) defined by $\left(G_{1}, \ldots, G_{n-1}\right)$. The points $Q_{1}, \ldots, Q_{r}$ of $S_{2}$ are in one-to-one correspondence with $P_{s+1}, \ldots, P_{s+r}$ 


\section{PURITy OF EXPONENTIAL SUMS ON $\mathbb{A}^{n}$}

via the inclusion $\mathbb{P}^{n-2}(\bar{k}) \hookrightarrow \mathbb{P}^{n-1}(\bar{k})$, and

$$
\begin{aligned}
\mathcal{O}_{S_{2}, Q_{i}} & =\mathcal{O}_{\mathbb{P}^{n-2}, Q_{i}} /\left(G_{1}, \ldots, G_{n-1}\right)=\mathcal{O}_{\mathbb{P}^{n-1}, P_{s+i}} /\left(F_{1}, \ldots, F_{n-1}, x_{n}\right) \\
& =\mathcal{O}_{\mathbb{P}^{n-1}, P_{s+i}} /\left(F_{1}, \ldots, F_{n-1}\right)=\mathcal{O}_{S_{1}, P_{s+i}},
\end{aligned}
$$

so the Milnor numbers are the same.

Now $G_{1}, \ldots, G_{n-1}$ fall under the hypotheses of the lemma, so we can apply the induction hypothesis and deduce that $\sum_{i=s+1}^{s+r} \nu_{i}=\left((d-1)^{n-1}-(-1)^{n-1}\right) / d$. Therefore,

$$
\sum_{i=1}^{s} \mu_{i}=\sum_{i=1}^{s} \nu_{i}=\sum_{i=1}^{s+r} \nu_{i}-\sum_{i=s+1}^{s+r} \nu_{i}=(d-1)^{n-1}-\left((d-1)^{n-1}-(-1)^{n-1}\right) / d=\left((d-1)^{n}-(-1)^{n}\right) / d .
$$

Thus, under the hypotheses of Theorem 11, $L\left(T, \mathcal{L}_{\psi(f)}\right)^{(-1)^{n+1}}$ is a polynomial of degree $(d-1)^{n}-\left(d-d^{\prime}\right)\left((d-1)^{n}-(-1)^{n}\right) / d=\left(d^{\prime}(d-1)^{n}+(-1)^{n}\left(d-d^{\prime}\right)\right) / d$.

\section{End of the proof of Theorem 2}

Part (3) of the theorem is a direct consequence of the previous two parts via the trace formula and Deligne's theorem. So it suffices to prove parts (1) and (2). Fix a positive integer $d^{\prime}<d$ prime to $p$ such that $d^{\prime} / d>p /\left(p+(p-1)^{2}\right)$. Denote by $\mathcal{P}_{d, d^{\prime}}$ the affine space of all polynomials in $k\left[x_{1}, \ldots, x_{n}\right]$ of degree $\leqslant d$ whose homogeneous component of degree $i$ is zero for all $d^{\prime}<i<d$. Let $\pi_{1}: \mathcal{P}_{d, d^{\prime}} \times \mathbb{A}_{k}^{n} \rightarrow \mathcal{P}_{d, d^{\prime}}$ be the projection and ev $: \mathcal{P}_{d, d^{\prime}} \times \mathbb{A}_{k}^{n} \rightarrow \mathbb{A}_{k}^{1}$ the evaluation map. Let $K \in \mathcal{D}_{c}^{b}\left(\mathcal{P}_{d, d^{\prime}}, \overline{\mathbb{Q}}_{\ell}\right)$ be the object $\mathrm{R} \pi_{1 !} e v^{\star} \mathcal{L}_{\psi}\left[n+\operatorname{dim} \mathcal{P}_{d, d^{\prime}}\right]$.

Lemma 13. The object $K$ is perverse and pure of weight $n+\operatorname{dim} \mathcal{P}_{d, d^{\prime}}$.

Proof. For $d^{\prime}=d-1$ (i.e. when $\mathcal{P}_{d, d^{\prime}}$ is the affine space of all polynomials of degree $\leqslant d$ ) this is [Kat05, Theorem 3.1.2(1)]. We will see that the same proof works in general.

There is a natural finite map $\tau: \mathbb{A}_{k}^{n} \rightarrow \hat{\mathcal{P}}_{d, d^{\prime}}$. Namely, for every $t \in \mathbb{A}^{n}(\bar{k}), \tau(t) \in \hat{\mathcal{P}}_{d, d^{\prime}}(\bar{k})$ is the evaluation map at $t, e v(-, t): \mathcal{P}_{d, d^{\prime}}(\bar{k}) \rightarrow \bar{k}$. As $\overline{\mathbb{Q}}_{\ell}[n]$ is perverse and pure of weight $n$ on $\mathbb{A}_{k}^{n}$, so is $\tau_{\star} \overline{\mathbb{Q}}_{\ell}[n]$ on $\hat{\mathcal{P}}_{d, d^{\prime}}$. Its Fourier transform $T_{\psi}\left(\tau_{\star} \overline{\mathbb{Q}}_{\ell}[n]\right) \in \mathcal{D}_{c}^{b}\left(\mathcal{P}_{d, d^{\prime}}, \overline{\mathbb{Q}}_{\ell}\right)$ with respect to $\psi$ is $K$ (by the very definition of $K$ ). Therefore, $K$ is perverse and pure of weight $n+\operatorname{dim} \mathcal{P}_{d, d^{\prime}}$ (cf. [KL85, §2], or [KW01, § III.8], for the definition and main properties of the Fourier transform).

Note that for every finite extension $k^{\prime} / k$ and every $f \in \mathcal{P}_{d, d^{\prime}}\left(k^{\prime}\right)$, the trace of the geometric Frobenius element in $\operatorname{Gal}\left(\bar{k} / k^{\prime}\right)$ acting on the stalk of $K$ at a geometric point over $f$ is the sum

$$
(-1)^{n+\operatorname{dim} \mathcal{P}_{d, d^{\prime}}} \sum_{x \in k^{\prime n}} \psi\left(\operatorname{Trace}_{k^{\prime} / k} f(x)\right)
$$

Let $U \subset \mathcal{P}_{d, d^{\prime}}$ be the maximal dense open set on which $K$ has lisse cohomology sheaves. Then $\mathcal{H}^{i}(K)_{\mid U}=0$ for $i \neq-\operatorname{dim} \mathcal{P}_{d, d^{\prime}}$ and $\mathcal{F}:=\mathcal{H}^{-\operatorname{dim} \mathcal{P}_{d, d^{\prime}}}(K)=\mathrm{R}^{n} \pi_{1 !} e v^{\star} \mathcal{L}_{\psi}$ is lisse and pure of weight $n$ on $U$. Thus, for different finite extensions $k^{\prime} / k$ and polynomials $f \in U\left(k^{\prime}\right)$, the exponential sums $\sum_{x \in k^{\prime n}} \psi\left(\operatorname{Trace}_{k^{\prime} / k} f(x)\right)$ are pure of weight $n$ and the same rank as $\mathcal{F}$.

Let $V \subset \mathcal{P}_{d, d^{\prime}}$ (respectively $W \subset \mathcal{P}_{d, d^{\prime}}$ ) be the dense open set of all polynomials $f$ such that $f_{d}$ defines a non-singular hypersurface on $\mathbb{P}_{\bar{k}}^{n-1}$ (respectively the dense open set of all $f$ such that $\partial f_{d} / \partial x_{1}, \ldots, \partial f_{d} / \partial x_{n}$ have a finite number of common zeroes in $\mathbb{P}_{\bar{k}}^{n-1}$ and the hypersurface $f_{d^{\prime}}=0$ does not contain any of them). We know that:

(i) for every $f \in V(k)$, we have $\mathrm{H}_{c}^{i}\left(\mathbb{A}_{\bar{k}}^{n}, \mathcal{L}_{\psi(f)}\right)=0$ for $i \neq n, n+1$; for $i>n+1$, this is Proposition 5 ; for $i<n$ it is just Poincaré duality, as $\mathbb{A}_{\bar{k}}^{n}$ is smooth and $\mathcal{L}_{\psi(f)}$ is lisse; 


\section{A. ROJAS-LEÓN}

(ii) for every $f \in W(k)$, the $L$-function

$$
L\left(T, \mathcal{L}_{\psi(f)}\right)^{(-1)^{n+1}}=\prod_{i=0}^{2 n} \operatorname{det}\left(1-T \cdot F \mid \mathrm{H}_{c}^{i}\left(\mathbb{A}_{\bar{k}}^{n}, \mathcal{L}_{\psi(f)}\right)\right)^{(-1)^{n+i}}
$$

is a polynomial of degree $N:=\left(d^{\prime}(d-1)^{n}+(-1)^{n}\left(d-d^{\prime}\right)\right) / d($ cf. $\S 3)$.

Recall that a constructible $\overline{\mathbb{Q}}_{\ell}$-sheaf $\mathcal{G}$ on a smooth connected scheme $S$ is said to be of perverse origin if there is a perverse sheaf $L \in \mathcal{D}_{c}^{b}\left(S, \overline{\mathbb{Q}}_{\ell}\right)$ such that $\mathcal{G}=\mathcal{H}^{-\operatorname{dim} S}(L)$ (cf. [Kat03, $\left.\S 1\right]$ ). In that case, we have the following (cf. [Kat03, Proposition 12]).

THEOREM 14. The integer valued function defined by $s \mapsto \operatorname{rank} \mathcal{G}_{\bar{s}}$ on $S$ (where $\bar{s}$ is a geometric point over $s$ ) is lower semicontinuous. In other words, the rank of $\mathcal{G}$ does not increase under specialization. In particular, the dimension of the stalk of $\mathcal{G}$ at any geometric point of $S$ can never exceed the generic rank of $\mathcal{G}$. Moreover, the largest open set on which $\mathcal{G}$ is lisse is precisely the set where the rank of $\mathcal{G}$ is maximal (equal to the generic rank).

Note that on $U$ the degree of the $L$-function is just the rank of $\mathcal{F}$. Therefore, on $U \cap W, \mathcal{F}$ is lisse of rank $N$. In particular, the generic rank of $\mathcal{F}$ is $N$. As $\mathcal{F}$ is of perverse origin, from Theorem 14 we deduce that for every $f \in \mathcal{P}_{d, d^{\prime}}(k)$ the cohomology group $\mathrm{H}_{c}^{n}\left(\mathbb{A}_{\bar{k}}^{n}, \mathcal{L}_{\psi(f)}\right)$ (which is the stalk of $\mathcal{F}$ at a geometric point over $f$ ) has dimension at most $N$.

Now let $f \in V \cap W(k)$. From part (i) we have

$$
L\left(T, \mathcal{L}_{\psi(f)}\right)^{(-1)^{n+1}}=\frac{\operatorname{det}\left(1-T \cdot F \mid \mathrm{H}_{c}^{n}\left(\mathbb{A}_{\bar{k}}^{n}, \mathcal{L}_{\psi(f)}\right)\right)}{\operatorname{det}\left(1-T \cdot F \mid \mathrm{H}_{c}^{n+1}\left(\mathbb{A}_{\bar{k}}^{n}, \mathcal{L}_{\psi(f)}\right)\right)} .
$$

On the other hand, from part (ii) we know that this is a polynomial of degree $N$. Therefore,

$$
\operatorname{dim} \mathrm{H}_{c}^{n}\left(\mathbb{A}_{\bar{k}}^{n}, \mathcal{L}_{\psi(f)}\right)-\operatorname{dim} \mathrm{H}_{c}^{n+1}\left(\mathbb{A}_{\bar{k}}^{n}, \mathcal{L}_{\psi(f)}\right)=N .
$$

As $\operatorname{dim} \mathrm{H}_{c}^{n}\left(\mathbb{A}_{\bar{k}}^{n}, \mathcal{L}_{\psi(f)}\right) \leqslant N$ and $\operatorname{dim} \mathrm{H}_{c}^{n+1}\left(\mathbb{A}_{\bar{k}}^{n}, \mathcal{L}_{\psi(f)}\right)$ cannot be negative, we conclude that $\mathrm{H}_{c}^{n}\left(\mathbb{A}_{\bar{k}}^{n}\right.$, $\left.\mathcal{L}_{\psi(f)}\right)$ has dimension $N$ and the group $\mathrm{H}_{c}^{n+1}\left(\mathbb{A}_{\bar{k}}^{n}, \mathcal{L}_{\psi(f)}\right)$ vanishes.

From Theorem 14 we deduce that $\mathcal{F}$ is lisse on $V \cap W$, as it has maximal rank there. Furthermore, it is pure of weight $n$, because $K_{\mid V \cap W}=\mathcal{F}_{\mid V \cap W}\left[\operatorname{dim} \mathcal{P}_{d, d^{\prime}}\right]$ is pure of weight $n+\operatorname{dim} \mathcal{P}_{d, d^{\prime}}$. This completes the proof of Theorem 2.

Remark 15. When $p=d=2$ and $n$ is even, the sum $\sum_{x \in k^{n}} \psi(f(x))$ is known to be pure of weight $n$ and rank 1 if the hypersurface defined by $f_{2}=0$ is non-singular (cf. [AS00a, §6]).

Remark 16. We will see now that, for the rank formula in Theorem 2 to hold, the restriction $d^{\prime} / d>p /\left(p+(p-1)^{2}\right)$ (or at least some milder lower bound for $d^{\prime}$ ) is essential. More precisely, let $d=p^{a} d_{0}$, where $d_{0}$ is prime to $p$. We claim that the formula is not true for $d^{\prime}<d_{0}$. Let $\mathcal{P}_{d,-1}$ be the affine space of homogeneous polynomials of degree $d$. Let $A$ (respectively $B$ ) be the generic rank of $\mathrm{R}^{n} \pi_{1 !} e v^{\star} \mathcal{L}_{\psi}$ on $\mathcal{P}_{d,-1}$ (respectively $\mathcal{P}_{d, d^{\prime}}$ ). By [Kat05, Theorem 3.6.5] we know that

$$
A=\frac{(d-1)^{n}+(-1)^{n}(d-1)}{d}+\frac{d_{0}-1}{d}\left((d-1)^{n}-(-1)^{n}\right) .
$$

On the other hand, as $\mathcal{P}_{d,-1} \subset \mathcal{P}_{d, d^{\prime}}$ and $\mathrm{R}^{n} \pi_{1 !} e v^{\star} \mathcal{L}_{\psi}$ is of perverse origin, we have the inequality $A \leqslant B$. However, it is easy to see that the inequality $A \leqslant\left(d^{\prime}(d-1)^{n}+(-1)^{n}\left(d-d^{\prime}\right)\right) / d$ is equivalent to $d_{0} \leqslant d^{\prime}$. Therefore, if $d^{\prime}<d_{0}$, we cannot have $B=\left(d^{\prime}(d-1)^{n}+(-1)^{n}\left(d-d^{\prime}\right)\right) / d$.

\section{Proof of Theorem 4}

We will use a similar procedure to prove the second result, therefore we will first show the following. 


\section{PURITY OF EXPONENTIAL SUMS ON $\mathbb{A}^{n}$}

Proposition 17. Suppose that $d$ is prime to $p$ and the hypersurface defined in $\mathbb{P}_{\bar{k}}^{n-1}$ by the equation $f_{d}=0$ has at worst isolated singularities. Then $\mathrm{H}_{c}^{i}\left(\mathbb{A}_{\bar{k}}^{n}, \mathcal{L}_{\psi(f)}\right)=0$ for $i>n+1$.

Proof. This is already proven, although not explicitly stated, in [Kat99, Theorem 16]. Let $\tilde{X}$ be the incidence variety defined in $\mathbb{P}_{k}^{n} \times \mathbb{A}_{k}^{1}$ (with coordinates $X_{0}, X_{1}, \ldots, X_{n}$ for the first factor and $\lambda$ for the second factor) by the vanishing of $F-\lambda X_{0}^{d}$, where $F$ is again the homogenization of $f$ with respect to the variable $X_{0}$. Let $\tilde{f}: \tilde{X} \rightarrow \mathbb{A}_{k}^{1}$ be the restriction of the second projection. The affine space $\mathbb{A}_{k}^{n}$ can be naturally embedded as a dense open subset of $\tilde{X}$ and, as in Lemma 7 , there is a quasi-isomorphism

$$
\mathrm{R} \Gamma_{c}\left(\mathbb{A}_{\bar{k}}^{n}, \mathcal{L}_{\psi(f)}\right) \stackrel{\sim}{\rightarrow} \mathrm{R} \Gamma_{c}\left(\tilde{X} \otimes \bar{k}, \mathcal{L}_{\psi(f)}\right)
$$

where we also denote by $\mathcal{L}_{\psi(f)}$ the pull-back of $\mathcal{L}_{\psi}$ to $\tilde{X}$ by $\tilde{f}$. The proof of [Kat99, Theorem 16], applied to $X=\mathbb{P}_{k}^{n}, L=X_{0}$ and $H=F$ (hence $\delta=0, \varepsilon=-1$ ) shows that

$$
\mathrm{H}_{c}^{a}\left(\mathbb{A}_{\bar{k}}, \mathrm{R}^{b} \tilde{f}_{\star} \overline{\mathbb{Q}}_{\ell} \otimes \mathcal{L}_{\psi}\right)=0
$$

for $a+b \geqslant n+2$. In particular, the spectral sequence

$$
\mathrm{H}_{c}^{a}\left(\mathbb{A}_{\bar{k}}, \mathrm{R}^{b} \tilde{f}_{\star} \overline{\mathbb{Q}}_{\ell} \otimes \mathcal{L}_{\psi}\right) \Rightarrow \mathrm{H}_{c}^{a+b}\left(\tilde{X} \otimes \bar{k}, \mathcal{L}_{\psi(f)}\right)
$$

implies that $\mathrm{H}_{c}^{i}\left(\mathbb{A}_{\bar{k}}^{n}, \mathcal{L}_{\psi(f)}\right) \cong \mathrm{H}_{c}^{i}\left(\tilde{X} \otimes \bar{k}, \mathcal{L}_{\psi(f)}\right)=0$ for $i>n+1$.

We will think of the homogeneous form $f_{d}$ and the integer $d^{\prime}$ as being fixed, and the degree $\leqslant d^{\prime}$ part of $f$, which we will call $g$, as being variable. Let $\mathcal{P}_{d^{\prime}}$ be the affine space of all polynomials of degree $\leqslant d^{\prime}$. Let $\pi_{1}: \mathcal{P}_{d^{\prime}} \times \mathbb{A}_{k}^{n} \rightarrow \mathcal{P}_{d^{\prime}}$ be the projection and $e v_{f_{d}}: \mathcal{P}_{d^{\prime}} \times \mathbb{A}_{k}^{n} \rightarrow \mathbb{A}_{k}^{1}$ the map $(g, x) \mapsto f_{d}(x)+g(x)$. Let $K \in \mathcal{D}_{c}^{b}\left(\mathcal{P}_{d^{\prime}}, \overline{\mathbb{Q}}_{\ell}\right)$ be the object $\mathrm{R} \pi_{1 !} e v_{f_{d}}^{\star} \mathcal{L}_{\psi}\left[n+\operatorname{dim} \mathcal{P}_{d^{\prime}}\right]$. Exactly as in Lemma 13, one shows the following.

Lemma 18. The object $K$ is perverse and pure of weight $n+\operatorname{dim} \mathcal{P}_{d^{\prime}}$.

For every finite extension $k^{\prime} / k$ and every $g \in \mathcal{P}_{d^{\prime}}\left(k^{\prime}\right)$, the trace of the geometric Frobenius element of $\operatorname{Gal}\left(\bar{k} / k^{\prime}\right)$ acting on the stalk of $K$ at a geometric point over $g$ is the sum

$$
(-1)^{n+\operatorname{dim} \mathcal{P}_{d^{\prime}}} \sum_{x \in k^{\prime n}} \psi\left(\operatorname{Trace}_{k^{\prime} / k}\left(f_{d}(x)+g(x)\right)\right) .
$$

Now let $V \subset \mathcal{P}_{d^{\prime}}$ be the open set of all polynomials $g$ whose homogeneous component of degree $d^{\prime}$ is non-zero and the hypersurface it defines in $\mathbb{P}_{\bar{k}}^{n-1}$ does not contain any of the singularities of $f_{d}=0$. For every $g \in V(k)$ we have $\mathrm{H}_{c}^{i}\left(\mathbb{A}_{\bar{k}}, \mathcal{L}_{\psi\left(f_{d}+g\right)}\right)=0$ for $i \neq n, n+1$, by Proposition 17 and Poincaré duality. On the other hand, by [AS00b, Theorem 1.10 and Proposition 6.5] we know that

$$
L\left(T, \mathcal{L}_{\psi\left(f_{d}+g\right)}\right)^{(-1)^{n+1}}=\frac{\operatorname{det}\left(1-T \cdot F \mid \mathrm{H}_{c}^{n}\left(\mathbb{A}_{\bar{k}}^{n}, \mathcal{L}_{\psi\left(f_{d}+g\right)}\right)\right)}{\operatorname{det}\left(1-T \cdot F \mid \mathrm{H}_{c}^{n+1}\left(\mathbb{A}_{\bar{k}}^{n}, \mathcal{L}_{\psi\left(f_{d}+g\right)}\right)\right)}
$$

is a polynomial of degree $N^{\prime}:=(d-1)^{n}-\left(d-d^{\prime}\right) \sum_{i=1}^{s} \mu_{i}$.

Let $U \subset \mathcal{P}_{d^{\prime}}$ be a dense open subset where $K$ has lisse cohomology sheaves. Then $\mathcal{H}^{i}(K)_{\mid U}=0$ for $i \neq-\operatorname{dim} \mathcal{P}_{d^{\prime}}$ and $\mathcal{F}:=\mathcal{H}^{-\operatorname{dim}} \mathcal{P}_{d^{\prime}}(K)=\mathrm{R}^{n} \pi_{1 !} e v_{f_{d}}^{\star} \mathcal{L}_{\psi}$ is lisse of rank $N^{\prime}$ and pure of weight $n$ on $U$. Being of perverse origin, by Theorem 14 this implies that for any $g \in \mathcal{P}_{d^{\prime}}(k)$ the cohomology group $\mathrm{H}_{c}^{n}\left(\mathbb{A}_{\bar{k}}^{n}, \mathcal{L}_{\psi\left(f_{d}+g\right)}\right)$ has dimension at most $N^{\prime}$. Moreover, if $g \in V(k)$, as

$$
\operatorname{dim} \mathrm{H}_{c}^{n}\left(\mathbb{A}_{\bar{k}}^{n}, \mathcal{L}_{\psi\left(f_{d}+g\right)}\right)-\operatorname{dim} \mathrm{H}_{c}^{n+1}\left(\mathbb{A}_{\bar{k}}^{n}, \mathcal{L}_{\psi\left(f_{d}+g\right)}\right)=N^{\prime}
$$

we conclude that $\mathrm{H}_{c}^{n+1}\left(\mathbb{A}_{\bar{k}}^{n}, \mathcal{L}_{\psi\left(f_{d}+g\right)}\right)=0$ and $\mathrm{H}_{c}^{n}\left(\mathbb{A}_{\bar{k}}^{n}, \mathcal{L}_{\psi\left(f_{d}+g\right)}\right)$ has dimension $N^{\prime}$. In particular, $\mathcal{F}_{\mid V}$ has constant rank $N^{\prime}$, so it is lisse by Theorem 14 . Therefore, as $K_{\mid V}=\mathcal{F}_{\mid V}\left[\operatorname{dim} \mathcal{P}_{d^{\prime}}\right]$ and $K$ is pure of weight $n+\operatorname{dim} \mathcal{P}_{d^{\prime}}$, the sheaf $\mathcal{F}$ must be pure of weight $n$ on $V$. This concludes the proof of Theorem 4. 


\section{PURITY OF EXPONENTIAL SUMS ON $\mathbb{A}^{n}$}

\section{ACKNOWLEDGEMENTS}

I would like to thank Nicholas Katz and Steven Sperber for their help and their useful comments and suggestions about some of the results in this article.

\section{REFERENCES}

AS00a A. Adolphson and S. Sperber, Exponential sums on $\mathbb{A}^{n}$, Israel J. Math. 120 (2000), 3-21.

AS00b A. Adolphson and S. Sperber, Exponential sums on $\mathbb{A}^{n}$, III, Manuscripta Math. 102 (2000), 429-446.

Del74 P. Deligne, La conjecture de Weil I, Publ. Math. Inst. Hautes Études Sci. 43 (1974), 273-307.

Del80 P. Deligne, La Conjecture de Weil II, Publ. Math. Inst. Hautes Études Sci. 52 (1980), 137-252.

Gar98 R. García López, Exponential sums and singular hypersurfaces, Manuscripta Math. 97 (1998), $45-58$.

Har77 R. Hartshorne, Algebraic geometry, Graduate Texts in Mathematics, vol. 52 (Springer, Berlin, 1977).

Kat93 N. Katz, Affine cohomological transforms, perversity, and monodromy, J. Amer. Math. Soc. 6 (1993), 149-222.

Kat99 N. Katz, Estimates for 'singular' exponential sums, Int. Math. Res. Not. (1999), 875-899.

Kat03 N. Katz, A semicontinuity result for monodromy under degeneration, Forum Math. 15 (2003), 191-200.

Kat05 N. Katz, Moments, monodromy, and perversity: a Diophantine perspective, Annals of Mathematics Studies, vol. 159 (Princeton University Press, Princeton, NJ, 2005).

KL85 N. Katz and G. Laumon, Transformation de Fourier et majoration de sommes exponentielles, Publ. Math. Inst. Hautes Études Sci. 62 (1985), 361-418.

KW01 R. Kiehl and R. Weissauer, Weil conjectures, perverse sheaves and l'adic Fourier transform, Ergebnisse der Mathematik und ihrer Grenzgebiete (3), vol. 42 (Springer, Berlin, 2001).

SGA4 P. Deligne, Application de la formule des traces aux sommes trigonométriques, in Cohomologie étale,

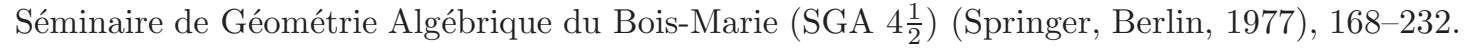

SGA7 A. Grothendieck, M. Raynaud, P. Deligne and D. Rim, Groupes de monodromie en géometrie algébrique, Séminaire de Géométrie Algébrique du Bois-Marie 1967-1969 (SGA 7 I), Lecture Notes in Mathematics, vol. 288 (Springer, Berlin, 1972).

Antonio Rojas-León arojasI@math.uci.edu

University of California, Irvine, Department of Mathematics, Irvine, CA 92697, USA 